\title{
La administración prehospitalaria de trombolíticos reduce la mortalidad en la fase aguda de un Infarto
}

Mortality and Prehospital Thrombolysis for Acute Myocardial Infarction. Morrison L., Verbeek P., McDonald A. Et al.J AMA.2000;283:26862692.

\section{Objetivo}

Evaluar la mortalidad asociada al uso de trombolisis prehospitalaria vs hospitalaria en pacientes con IAM.

\section{Diseño}

Revisión bibliográfica y de los principales estudios multicéntricos (1982-1999) realizada por expertos utilizando métodos y técnicas fundamentadas en Metaanálisis.

\section{Selección de estudios}

Inicialmente se incluyeron todos los estudios randomizados y controlados de Trombolisis Prehospitalaria vs Hospitalaria en Infarto Agudo con medición de mortalidad total en fase hospitalaria. Luego de un análisis exhaustivo, solo 6 de esos estudios ingresaron en este metaanálisis por cumplir los criterios de inclusión.

\section{Extracción de datos}

Fue realizada por 2 expertos en forma ciega. La calidad de los estudios analizados fue revisada en forma independiente por otros 2 coautores, también en forma ciega

\section{Resultados Principales}

Los resultados de los 6 (seis) ensayos randomizados $(n=6434)$ indican una reducción significativa de la mortalidad total en fase hospitalaria en aquellos pacientes tratados con trombolíticos prehospitalarios comparados con los que recibieron los trombolíticos en el hospital (odds ratio, 0.83; IC del 95\%, 0.70-0.98).

Los resultados fueron similares a pesar de diferentes calidades de estudios o del entrenamiento y la experiencia del administrador de la droga en cuestión.

El tiempo estimado hasta recibir el trombolítico fue 104 minutos en el grupo prehospitalario y 162 minutos para aquellos pacientes que se trombolizaron en el hospital ( $p: 0.007$ ).

\section{Conclusiones}

Este metaanálisis sugiere que la administración de tromboliticos en fase prehospitalaria para pacientes que cursan un IAM disminuye significativamente los tiempos hasta la administración de la droga y lo que es más importante, la mortalidad total (cardiovascular y no cardiovascular) durante la internación.

\section{Comentario}

Cerca del $40 \%$ de los pacientes que sufren un IAM fallecen antes de llegar al hospital como consecuencia de las complicaciones eléctricas de la oclusión coronaria aguda.

Por otra parte, en todos los estudios de reperfusión en el IAM la precocidad del tratamiento fue más importante que el tipo de estrategia empleada, con diferencias muy significativas en la reducción de la mortalidad de hasta el $50 \%$ en los casos tratados dentro de la hora de inicio de los síntomas.

En nuestro medio no existe experiencia randomizada sobre e tratamiento trombolítico prehospitalario versus hospitalario y este metaanálisis es de vital importancia para conocer la experiencia internacional en este tema.

Los 3 estudios más importantes, GREAT y EMIP (europeos) y MITI 1,2, de Estados Unidos, incluyeron gran cantidad de pacientes tratados con Tpa, un grupo en la fase prehospitalaria y otro en el hospital. Los resultados de estos estudios mostraron que: - Hay solo una tendencia (no estadísticamente significativa) en la reducción de la mortalidad a favor de administración prehospitalaria. - Se acortó el tiempo al tratamiento trombolítico tanto en los pa- cientes tratados en el domicilio como también en el hospital al reducir la demora entre la llegada al hospital y el tratamiento correspondiente.

- Los pacientes tratados en la fase prehospitalaria en forma precoz (antes de los 70 minutos) mostraron evidencias de infartos menores, mejor función ventricular y mayor sobrevida.

De todas maneras, y de acuerdo con las posibilidades de cada región, es indispensable implementar medidas para que el tratamiento de reperfusión comience dentro de los 90 minutos, para lo cual se deberá actuar en los diferentes puntos de demora: demora del paciente en consultar, demora del traslado hasta el centro asistencial y demora en el centro asistencial hasta la implementación efectiva del tratamiento.

No olvidemos que este planteo se encuentra con la dificultad para la selección de los pacientes apropiados, dado que requiere de una fuerte inversión y entrenamiento de los sistemas de emergencias, dificultad aún mayor en países como el nuestro, con escasos y mal administrados recursos económicos para la salud.

\section{Dr. Pablo García Marletti [ Servicio de Cardiología. Hospital Italiano de Bs. As. ]}

\section{Referencias}

1- Quantification of the benefit of earlier thrombolytic therapy: five-year results of the Grampian Region Early Anistreplase Trial (GREAT). J Am Coll Cardiol. 1997 Nov 1;30(5):1181-6. 2- Influence of early prehospital thrombolysis on mortality and event-free survival (the Myocardial Infarction Triage and Intervention [MITI] Randomized Trial). MITI Project Investigators. Am J Cardiol. 1996 Sep 1;78(5):497-502. 